\title{
Biosensors for Aflatoxins Detection
}

\author{
Lucia Mosiello and Ilaria Lamberti \\ ENEA, Italian National Agency for New \\ Technologies, Energy and the Environment, Rome, \\ Italy
}

\section{Introduction}

The availability of rapid and reliable methods for rapid determination of small molecules, as contaminants in food samples, including Aflatoxins, is an increasing need also for human health. In order to monitoring food contaminants, as Mycotoxins (MTXs) Gas Chromatographic (GC) and High Pressure Liquid Chromatography (HPLC) methods are generally utilized, due to their high detection sensibility and selectivity. However, GC and HPLC analyses are time consuming and needs sample pre-treatment or pre-concentration procedures. Immunoassays and biosensors are becoming a recognized alternative or complementary to conventional analytical techniques for the detection of mycotoxins, as Aflatoxins.

Recently, biosensors based on the use of monoclonal or polyclonal antibodies have seen a great development in the field of small molecules analytical determination and specifically in the mycotoxins analyses. Among biosensors for mycotoxins monitoring, optical or electrochemical devices for Aflatoxins detection were described by different authors. The present Chapter describes the different biosensors for Aflatoxins developed and utilized in food analysis. The absence of cross-reactivity obtained with most of these biosensor, the possibility of on-line measurement, the absence of sample pre-treatment, can really put it in competition with other conventional systems such as HPLC and ELISA.

Chapter describes also main biosensors features and vantages for these innovative devices and various examples of biosensors and reviews some biosensors for Aflatoxins and other mycotoxins detection methods, as microarray.

In particular, we will focus our attention on biosensors developed for mycotoxins detection that utilize immunoglobulins or aptamer showing affinity for a correspondent analyte, associated to various transduction elements. Various biosensing platforms will be introduced, including, but not limited to, surface plasmon resonance and quartz microbalance crystals. Examples of biosensors array, as microarray, detecting Aflatoxin and Fumonisin will be also presented. Some of these biosensing devices were developed in our laboratories and the sensing performance of each device will be evaluated and compared in terms of sensitivity and detection limit.

Analytical methods used for mycotoxins determination are mainly based on TLC, HPLC or ELISA. Actually biosensor and microsystem technologies are used for different applications including studies of human and veterinary diseases, drug discovery, genetic screening, clinical and food diagnostics. According to these approaches the aim of many authors was to transfer the methods of the immunological assay from microtiter plates into a biosensor format in order to develop a rapid, sensitive and inexpensive method for the detection of 
mycotoxins for food safety applications. Microarray and biosensor technology enables the fast and parallel analysis of a multitude of biologically relevant parameters. Not only nucleic acid-based tests, but also peptide, enzyme and antibody assays using different formats of biosensor evolved within the last decade. Antibody-based microarrays are a powerful assay technology that can be used to generate rapid detection of analytes in complex samples which, in our opinion, is also potentially useful for the generation of rapid immunological assay of food contaminants.

\section{Aflatoxins}

Mycotoxins are secondary metabolites that moulds produce naturally. Due to their ubiquitous presence in foodstuffs and their potential risk for human health, prompt detection is important. It is estimated that approximately $25 \%$ of the world's crops are contaminated to some extent with mycotoxins. Some mycotoxins (e.g.,aflatoxins) have been designated biowarfare agents due to their potential carcinogenicity. (Prieto-Simón et al., 2007) .

Aflatoxins are highly toxic and carcinogenic secondary metabolites produced mainly by three anamorphic species of the genus Aspergillus: A. flavus, A. parasiticus and A. nomius (Ehrlich et al., 2003). They are the most potent, naturally-occurring carcinogens known and have been linked to liver cancer and several other maladies in animals and humans (Turner et al., 2003; Valdivia et al., 2001; Otim et al., 2005).

When aflatoxin B1 (AFB1), the most toxic aflatoxin, is ingested by cows through contaminated feed, it is transformed into aflatoxin M1 (AFM1) through enzymatic hydroxylation of AFB1 at the 9a-position (see below) and has an approximate overall conversion rate equal to 0.3 to $6.2 \%$.

AFM1 is secreted in milk by the mammary gland of dairy cows. Even though it is less toxic than its parent compound, AFM1 has hepatotoxic and carcinogenic effects. This toxin, initially classified as a Group 2B agent, has now been reclassified as Group 1 by the International Agency for the Research on Cancer (IARC).

Another important class of MTX are those produced by Fusarium moniliforme, a prevalent fungus that infects corn and other cereal grains.

Fumonisin $\mathrm{B}_{1}\left(\mathrm{FB}_{1}\right)$ is the most common mycotoxin produced by F. moniliforme, suggesting it has toxicologic significance. Ingestion of moldy corn infected by F. moniliforme or closely related fungi is linked to a higher incidence of primary liver cancer (Ueno et al., 1997) and esophageal cancer in regions of South Africa and China.

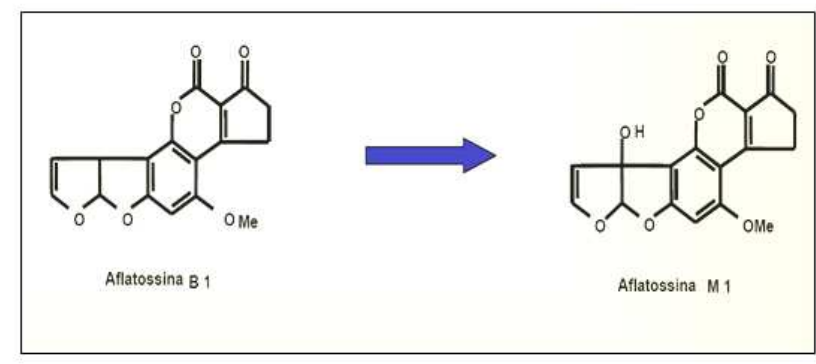

Aflatoxin molecules

Fig. 1. Aflatoxins B1 and M1 and Aspergillus fungus
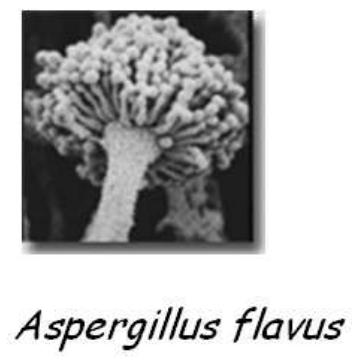


\section{Biosensors}

A biosensor is an analytical device for the detection of an analyte that combines a biological component with a physicochemical detector component. The most commune biosensor scheme is reported in Fig. 2 and it is consists of 3 parts:

- the sensitive biological element, or biological material (e.g. tissue, microorganisms, organelles, cell receptors, enzymes, antibodies, nucleic acids, etc.), a biologically derived material. The sensitive elements can be created by biological engineering.

- the transducer or the detector element (works in a physicochemical way; optical, piezoelectric, electrochemical, etc.) that transforms the signal resulting from the interaction of the analyte with the biological element into another signal (i.e., transducers) that can be more easily measured and quantified;

- $\quad$ associated electronics or signal processors that are primarily responsible for the display of the results in a user-friendly way.
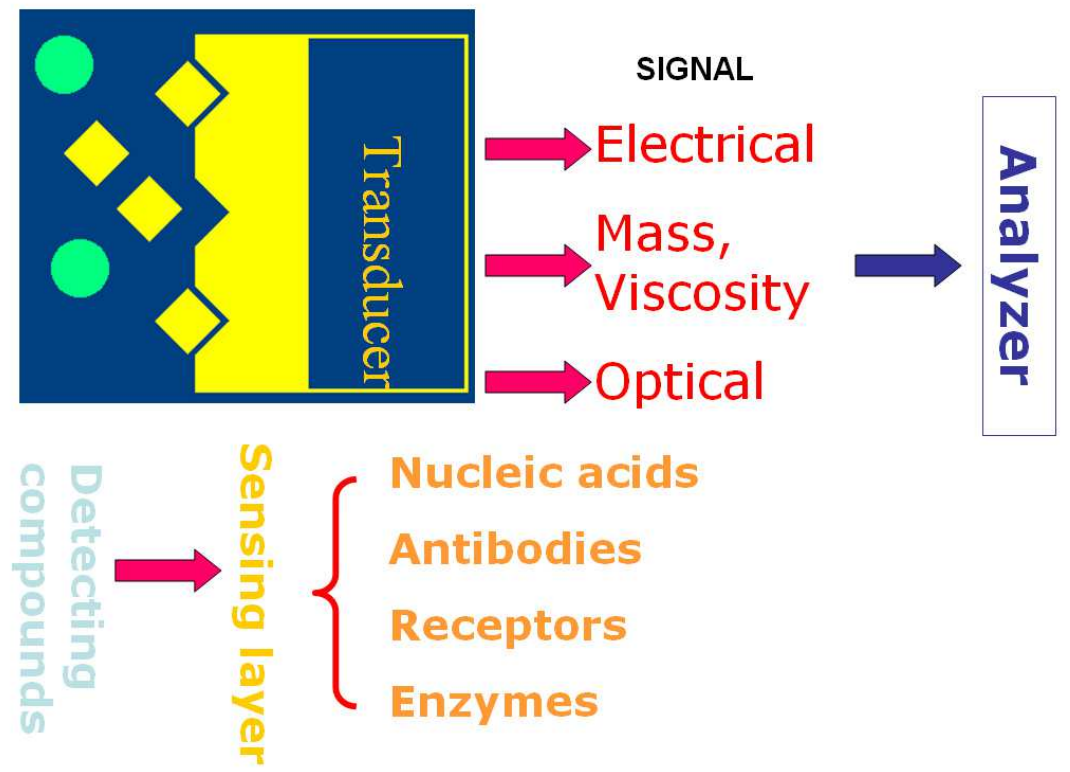

Fig. 2. Scheme of a Biosensor

Main advantages of biosensors technology in comparison with traditional analytical methods are fast detection (minutes) and response (seconds), high sensitivity (typically $\mathrm{nM}$, improved sensitivity with nanoparticles $\mathrm{pM}$ and better), their high selectivity, easy preparation and operation assay method. In addition most of these devices are reusable and show low cost assay (Typically less then $10 \mathrm{EUR} /$ sensor).

The methodology of surface chemistry is the basic knowhow for obtaining reproducible results with biosensors and various strategies can be used (Gagliardi et al, 2007).

The key points to consider when selecting an appropriate surface and coating procedure are a low degree of unspecific binding sites and uniform distribution of functional groups on the substrate surface. 
For this reason during biosensor development and testing particular attention would be focused on

- $\quad$ Surface (on wich sensing layer will be coated) characterisation

- Biological reagent (immunoglobulin, nucleic acid, ecc.) characterisation

- Uniformity of biological element

- Standard solution preparation

- Calibration and Standard Curve construction

Among biosensors piezo-electric devices are sensors that integrate a biological element with a physiochemical transducer to produce an electronic signal proportional to an analyte which is then conveyed to a detector.

Mass sensitive piezoelectric transducers are usually based on AT-cut quartz crystal covered by gold electrodes. The external alternating voltage induces oscillation of the quartz. The frequency of this oscillation depends on the transducer thickness (Fig.3).

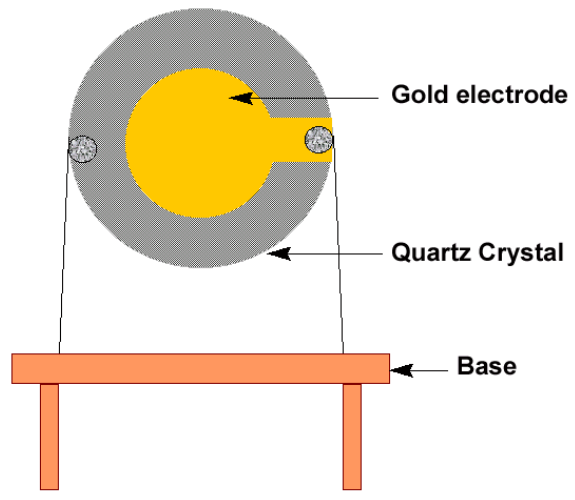

(a)

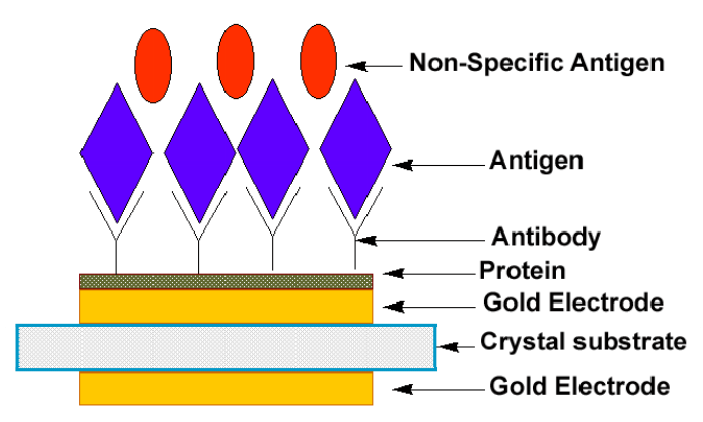

(b)

Fig. 3. (a) Mass piezoelectric trasducer; (b) A biosensor antibody-based

In these biosensors the frequency value of the oscillation of the quartz is proportional to the mass of the crystal following the Sauerbrey law and decreases with increasing of the mass (Equation 1, Sauerbrey equation).

$$
\Delta \mathrm{f}=-2.26 \times 10^{-6} \mathrm{f}_{0}{ }^{2}(\Delta \mathrm{m} / \mathrm{A})
$$

Responding to the need to achieve high sensitivity and move to the use of disposable probes, several electrochemical immunosensors have recently been reported in literature for the detection of AFB1 (Aflatoxin B1) in corn and barley and AFM1 (Aflatoxin M1) in milk. In particular, for AFB1 determination, an indirect competitive electrochemical immunoassay has been developed using disposable screen-printed carbon electrodes.

In an another work was presented a biosensing method for detection of aflatoxin $B_{1}$ and type-A trichothecenes, based on the use of indirect competitive ELISA format coupled with a 96-well screen-printed microplate.

Electrochemical immunoassays for $\mathrm{AFB}_{1}, \mathrm{~T}-2$, and $\mathrm{HT}-2$ were performed and the activity of the alkaline phosphatase or horseradish peroxidase labeled enzymes were measured using intermittent pulse amperometry (IPA) as electrochemical technique. Using standard 
solutions of the target analyte the LOD of the assays were 0.3 and $0.2 \mathrm{ng} \mathrm{ml}^{-1}$ for T-2 and $\mathrm{AFB}_{1}$ respectively, while the sensitivity was $1.2 \mathrm{ng} \mathrm{ml}^{-1}$ for both. For Aflatoxin $\mathrm{B}_{1}$, a stability study of electrochemical plate was also performed. Moreover, the matrix effect was evaluated using two different extraction treatments from corn.

The specificity of the assay was assessed by studying the cross-reactivity of the MAb (Monoclonal Antibody) towards other aflatoxins. The results indicated that the MAb could readily distinguish AFB1 from other toxins, with the exception of AFG1 (Piermarini, et al., 2007).

In the field of enzymatic/amperometric biosensor application an electrochemical immunosensor for the detection of ultratrace amounts of aflatoxin M1 (AFM1) in food products was developed.

This sensor was based on a competitive immunoassay using horseradish peroxidase (HRP) as a tag. Magnetic nanoparticles coated with antibody (anti-AFM1) were used to separate the bound and unbound fractions. The samples containing AFM1 were incubated with a fixed amount of antibody and tracer [AFM1 linked to HRP (conjugate)] until the system reached equilibrium. Competition occurs between the antigen (AFM1) and the conjugate for the antibody. Then, the mixture was deposited on the surface of screen-printed carbon electrodes, and the mediator [5-methylphenazinium methyl sulphate (MPMS)] was added.

The enzymatic response was measured amperometrically. A standard range $(0,0.005,0.01$, $0.025,0.05,0.1,0.25,0.3,0.4$ and $0.5 \mathrm{ppb}$ ) of AFM1-contaminated milk from the ELISA kit was used to obtain a standard curve for AFM1. To test the detection sensitivity of our sensor, samples of commercial milk were supplemented at $0.01,0.025,0.05$ or $0.1 \mathrm{ppb}$ with AFM1.

Immunosensor for Afla M1 described has a low detection limit (0.01 ppb), which is under the recommended level of AFM1 [0.05 $\mu \mathrm{g} \mathrm{L-1} \mathrm{(ppb)],} \mathrm{and} \mathrm{has} \mathrm{good} \mathrm{reproducibility.}$

Recently an innovative amperometric biosensor for AflatoxinB1 was described. This biosensor was developed using the enzyme conjugate aflatoxin-oxidase (AFO), embedded in sol-gel, linked to multiwalled carbon nanotubes (MWCNTs)-modified Pt electrode and was reported for the first time.

The covalent linkage between AFO and MWCNTs retained enzyme activity and responsed to the oxidation of afltoxin $\mathrm{B}_{1}\left(\mathrm{AFB}_{1}\right)$. Its apparent Michaelis-Menten constant for $\mathrm{AFB}_{1}$ was $7.03 \mu \mathrm{mol} \mathrm{L}^{-1}$, showing a good affinity. The sensor exhibited a linear range from 3.2 $\mathrm{nmol} \cdot \mathrm{L}^{-1}$ to $721 \mathrm{nmol} \cdot \mathrm{L}^{-1}(1 \mathrm{ng} / \mathrm{ml}$ to $225 \mathrm{ng} / \mathrm{ml})$ with limits of detection of $1.6 \mathrm{nmol} \cdot \mathrm{L}^{-1}$ (signal-to-noise ratio $=3$ ), an average response time of $44 \mathrm{~s}$ (less than $30 \mathrm{~s}$ when $\mathrm{AFB}_{1}$ Conc. is bigger than $45 \mathrm{ng} / \mathrm{ml}$ ), and a high sensitivity of $0.33 \times 10^{2} \mathrm{~A} \mathrm{~mol}^{-1} \cdot \mathrm{L} \mathrm{cm}^{-2}$. The active energy was $18.8 \mathrm{~kJ} \mathrm{~mol}^{-1}$, demonstrating the significant catalyzation of AFO for oxidation of $\mathrm{AFB}_{1}$ in this biosensor.

These results demonstrated that AFO act at the unsaturated carbon bond of bisfuran ring in $\mathrm{AFB}_{1}$, to primarily form an unstable compound: oxygen additive product and hydrogen peroxide. This makes a clear choice to use AFO as a recognition receptor for biosensors to detect this mycotoxin (Li et al., 2011).

\section{SPR biosensor for aflatoxins}

A promising technology for rapid Afaltoxins detection is the surface plasmon resonance biosensor. The principle of surface plasmon resonance is based on the detection of a change of the refractive index of the medium when an analyte binds to an immobilised partner molecule (antibody). 
Optical sensors based on excitation of surface plasmons, commonly referred to as surface plasmon resonance (SPR) sensors, belong to the group of refractometric sensing devices. Development of SPR sensors for detection of chemical and biological species has gained considerable momentum, and the number of publications reporting applications of SPR biosensors for detection of analytes related to medical diagnostics, environmental monitoring, and food safety and security has been rapidly growing.

SPR affinity biosensors are sensing devices which consist of a biorecognition element that recognizes and is able to interact with a selected analyte and an SPR transducer, which translates the binding event into an output signal. The biorecognition elements are immobilized in the proximity of the surface of a metal film supporting a surface plasmon.

Analyte molecules in a liquid sample in contact with the SPR sensor bind to the biorecognition elements, producing an increase in the refractive index at the sensor surface, which is optically measured.

The change in the refractive index produced by the capture of biomolecules depends on the concentration of analyte molecules at the sensor surface and the properties of the molecules. If the binding occurs within a thin layer at the sensor surface of thickness, the sensor response is proportional to the binding-induced refractive index change. (Homola, 2008). The SPR principle is reported in Fig.4.

These biosensors show several advantages such as small sample volumes ( $\mu \mathrm{L}$ volumes) and reusable metal chips.

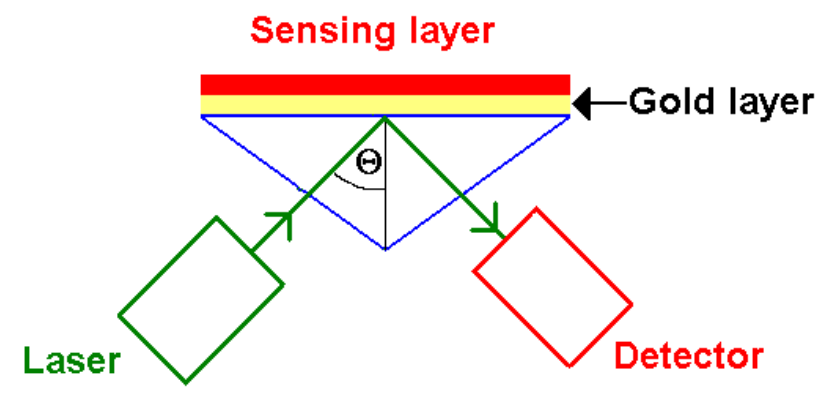

Fig. 4. SPR biosensor principle, surface plasmons are excited by polarised laser beam at certain angle $\Theta$ and the intensity of reflected light is measured.

Authors published data obtained using a SPR biosensor for Aflatoxin detection in maize extracts (Cuccioloni et al.).

In this paper different dilutions of Aflatoxin-containing and Aflatoxin-free fractions were added to the elastase-functionalized surface, and each response kinetic was routinely followed and analyzed as described above. The regeneration of the elastase monolayer was carried out as previously described. Detection procedures were replicated on different days on both the same and different elastase-functionalized surfaces. Additionally, the assessment of the number of regeneration cycles that a sensor surface can withstand without a significant loss of the sensitivity and accuracy of the assay and the stability of the sensing surface throughout multiple measurements were evaluated.

Limits of Detection and Quantitation: In compliance with the IUPAC rules, the limit of detection (LOD) was calculated as three times the standard deviation of the blank measurements. The limit of quantification (LOQ) is calculated as 10 times the standard 
deviation of the blank measurements. Aflatoxin-free certified T400A maize sample was used as a blank matrix.

Calibration Curve: Biosensor-based assay was applied for the determination of AFB1 using spiked maize samples (Figure 3 ). The analysis of the binding of AFB1 to elastase over the concentration range 1-50 $\mu \mathrm{g} / \mathrm{kg}$ reported that the response for the optimized assay was linear in the range between 1.67 and $17.8 \mu \mathrm{g} / \mathrm{kg}$. The calibration procedure was replicated on three different days. The experimentally measured lower limit of the linear range was $1.67 \mu \mathrm{g} / \mathrm{kg}$ of AFB1, whereas the KD was $0.91 \mu \mathrm{M}(\approx 250 \mu \mathrm{g} / \mathrm{kg})$ AFB1. The detection limits reached allow us to use this assay for detection of AFB1 in maize within the regulatory limits.

Recently some authors presented during a Nanotechology Conference a SPR biosensor for Aflatoxin B1 developed using fusion proteins as a linker.

Because one of the main goal in the development of SPR immunosensors is efficient immobilization of antibodies. Conventional methods, such as self-assembled monolayers (SAMs) of alkanethiols cause antibodies to be random oriented. To improve antibody linker and orientation in their work, the authors constructed a novel fusion protein by genetically fusing gold binding polypeptides (GBP) to protein A (ProA).

The resulting GBP-ProA protein was directly self-immobilized onto SPR gold chip surfaces via the GBP portion, followed by the oriented binding of anti-AFB 1 antibodies onto the ProA domain and $\mathrm{AFB}_{1}$ in series. Consequently, a low detection limit $(10 \mathrm{ng} / \mathrm{mL})$ has been achieved for mycotoxin SPR immunosensor by using GBP-ProA fusion proteins as a crosslinker. ( Ko et al., 2010).

\section{QMC biosensor for others mycotoxins}

A Quarz Crystal Microbalance (QCM) consists of a thin quartz disk with a electrodes plated. The application of an external electrical potential to a piezoelectric material produces internal mechanical stress. As the QCM is piezoelectric, an oscillating electric field applied across the device induces an acoustic wave that propagates through the crystal and meets minimum impedance when the thickness of the device is a multiple of a half wavelength of the acoustic wave. Deposition of a thin film on the crystal surface decreases the frequency in a portion to the mass of the film.

As described, the mycotoxins, such are Aflatoxins are toxical fungal metabolites that can occur in primary food products. In order to new biosensor development we focused our attention also on Ochratoxin A (OTA), which was discovered as a metabolite of Aspergillus Ochraceus (Van der Merwe et al., 1965). This mycotoxin generally appears during storage of cereals, coffee, cocoa, dried fruit, pork etc. and occasionally in the field of grapes. It may also be present in blood and kidneys of animals that have been fed on contaminated feeds. Animal studies indicated that this toxin is carcinogenic (Turner et al., 2009). Therefore, the European Commission has fixed maximum concentration of OTA in foodstuffs: $3 \mu \mathrm{g} / \mathrm{kg}(7.4$ $\mathrm{nM}$ ) for cereal products and $5 \mu \mathrm{g} / \mathrm{kg}(12.4 \mathrm{nM})$ for roasted coffee, respectively (Commission Regulation No. 1881/2006, 19 December 2006).

The establishment of efficient method of this analyte detection is therefore of high importance. In addition to traditional, but expensive and time-consuming methods such as liquid chromatography, new trends consist in development portable and easy to use biosensors (Siontorou et al. ,1998).

Most of the biosensors for this analyte detection developed so far were based on electrochemical detections such as oxidation of OTA at glassy carbon electrode (limit of 
detection (LOD) $0.26 \mu \mathrm{M}$ ) (Oliveira et al., 2007) or reduction of horseradish peroxidase (LOD $0.25 \mathrm{nM}$ ) (Alonso-Lomillo et al., 2010 ).

Immunosensor based on quartz crystal microbalance (QCM) was recently reported as well (Tsai et al.,2007). In this sensor anti-OTA antibodies were immobilised on a surface of 16mercaptohexadecanoic acid. The detection based on the competitive binding between free OTA and that conjugated with BSA provided LOD $40 \mathrm{nM}$.

Recently a DNA aptamer sensitive to OTA has been developed (Cruz-Aguado et al., 2008). This aptamer was able to recognize OTA with sensitivity in a ppb level and with high selectivity. The electrochemiluminiscence biosensor using aptamers as receptors was recently developed (LOD $17 \mathrm{pM}$ ).

Thus most of the biosensors for mycotoxin OTA reported were based on indirect detection methods. Would be, however, rather useful to develop biosensor based on direct method that do not require additional modification of receptor or complicated multi stage assay. In a recent work Prof.T.Hianik (Comenius University, Bratislava, Slovakia) made therefore attempt to develop biosensor for OTA based on thickness shear mode acoustic method (TSM) using biotinylated DNA aptamers immobilised on a surface of quartz crystal transducer covered by neutravidin (Lamberti et al. 2011) .

TSM is certain analogy of QCM, however, in addition to mass, the TSM determines also the viscosity contribution arising from the friction between biolayer and the surrounding buffer (Fig.5).

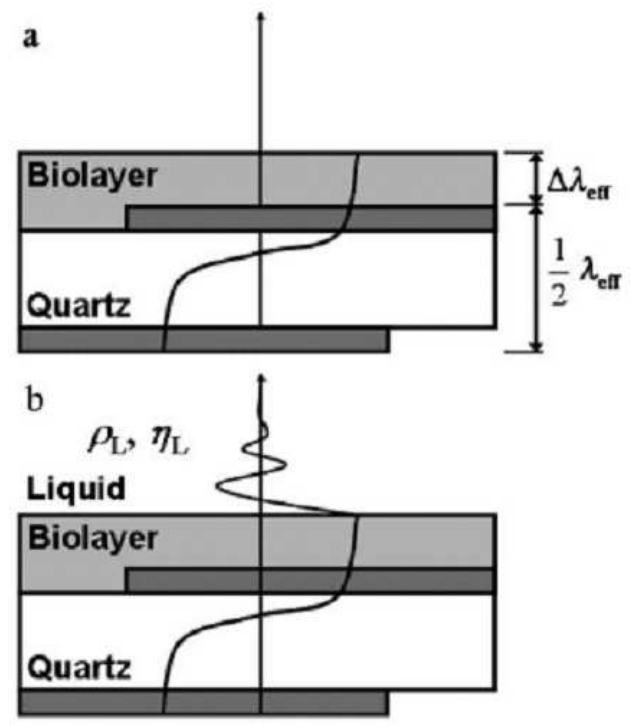

a) Biolayer in an air No energy dissipation into the surrounding environment. However, due to the viscoelasticity of biolayer, certain part of energy dissipates.

\section{b) Biolayer in liquid Substantial dissipation takes place due to viscosity effect.}

Fig. 5. Propagation of acoustic wave from the sensor surface

This is important for detection of small molecules, such are mycotoxins for which the QCM detection is difficult due to small molecular weight of the analyte. We showed that TSM allowing detecting this mycotoxin with LOD $30 \mathrm{nM}$ and with good selectivity. He also studied the stability of DNA aptamers depending on concentration of calcium ions, that are 
important for binding OTA to DNA aptamer. In our opinion the described biosensor would be applied also for Aflatoxins detection.

\section{Microarray for aflatoxin B1 detection}

Microarrays provide a powerful analytical tool for the simultaneous detection of multiple analytes in a single experiment and consist of a biosensor micro o nano arrays.

Research on microarrays as multianalyte biosystems has generated increased interest in the last decade. The main feature of the microarray technology is the ability to simultaneously detect multiple analytes in one sample by an affinity-binding event at a surface interface. In some cases immunoanalytical microarrays have the potential to replace conventional chromatographic techniques. They are applied if the number of samples is high or analysis by current methods is difficult and/or expensive. Therefore, microarray platforms have a great potential as monitoring systems for the rapid assessment of water or food samples. Antibody-based microarrays are a powerful tool for analytical purposes, also for Aflatoxins detection application. Immunoanalytical microarrays are a quantitative analytical technique using antibodies as highly specific biological recognition elements. They can be designed for a variety of analytical applications producing rapid results with low limits of detection (LOD).

For these reasons in association to some biosensors for Aflatoxins examples, we reported in this Chapter also a feasibility study, made in our laboratories, on application of antibodies microarrays for simultaneous analysis of two different mycotoxins (AflatoxinB1 and Fumonisin B1). In this work we developed a competitive immunoassay in a microarray format and with the described method observed different microarray patterns in samples containing Aflatoxin-B1 or Fumonisine or either analytes at a ppb concentration range (Lamberti et al., 2009). The quality of the microarray data is comparable to data generated by a microplate-based immunoassay (ELISA), but further investigations are needed in order to better characterize these methods when applied for food contaminants determination. In any case we hope that our results can confirm the feasibility to develop hapten microarrays as for the immunochemical analysis of mycotoxins, as above described for others small organic molecules (e.g. bacterial toxins or biological warfare agents).

Enzyme linked immunosorbent assay (ELISA) and fluorescence immunoassay (FIA) are excellent survey tools for many analitycal purpose because of their high-throughput, user friendliness, and field portability. These important characteristics make immunoassays attractive tools for food testing by regulatory agencies to ensure food safety. Immunoassay is traditionally performed as individual test, however in many cases it is necessary to perform a panel of tests on each sample (detection of drug residues). To address this requirement, microarray-based immunoassay technologies have been developing utilizing microarray platform (multianalyte analysis) and classic immunoassay (multi-samples analysis).

In recent years, the antibody microarray technology has made significant progress, going from proof -of-concept designs to established high-performing technology platforms capable of targeting non-fractionated complex samples, as proteoma (Blohm \& GuiseppiElie., 2001).

Microarrays consist of immobilized biomolecules spatially addressed on planar surfaces, microchannels or microwells, or an array of beads immobilized with different biomolecules. 
Biomolecules commonly immobilized on microarrays include oligonucleotides, polymerase chain reaction (PCR) products, proteins, lipids, peptides and carbohydrates. Ideally, the immobilized biomolecules must retain activity, remain stable, and not desorb during reaction and washing steps. The immobilization procedure must ensure that the biomolecules are immobilized at optimal density to the microarray surface for efficient binding (Venkatasubbarao, S. et al., 2004).

Some microarray applications are focused on current trends in the movement of this technology from being a purely research method to becoming an analytical instrument applicable in the clinic and as well as in human health (Koppal, T. , 2004).

According to this trend we have tried to transfer the immunoassay method from microtiter plates into a microarray format in order to develop a multiparametric, rapid, sensitive and inexpensive method for the the detection of mycotoxins for food safety application.

To perform our test and check the feasibility of this format, we focused our studies on the most popular mycotoxins Aflatoxin B1 (AFB1) and Fumonisin B1 (FB1) and developed a competitive immunoassay in a microarray format, using the Dr.Chip platform provided by Life Line Lab Co. (Pomezia, Italy) and used also for other applications (Lamberti, 2010).

Microarray platform is equipment to create microarrays and to read the final results, via densitometric detection, based on the enzymatic and colorimetric assay. In Fig.6 are reported a detail of the plastic probe tray for protein spotting and pins. In the same picture is also shown the scheme of the glass treated with functional protein linker.

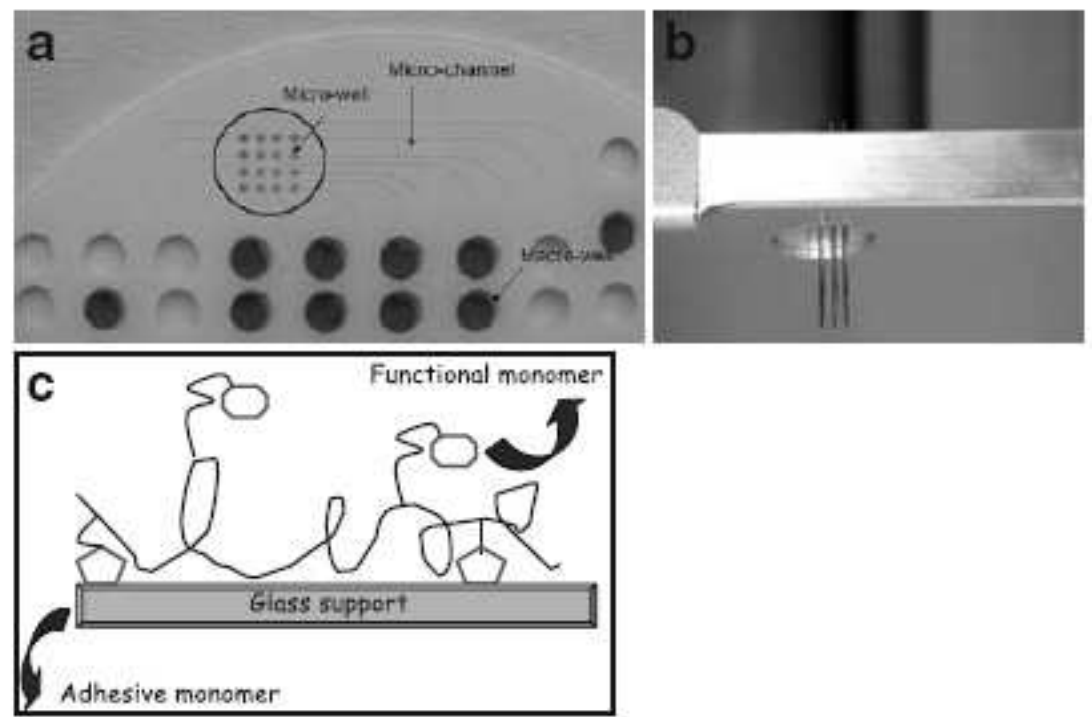

Fig. 6. Microarray spotting platform used for Aflatoxin B1 detection

As in other conventional competitive immunoassay the color intensity and corrispondent grey values obtained from antigen microarrays BSA-Afla B1, prepared as described in this paper and used in our immunological tests, are in inverse proportion to antigen concentration in standard solutions. Assay method for Aflatoxin is described in Fig.7. 


\section{SUMMARY OF ANTIBODY MICROARRAY OF MYCOTOXIN}

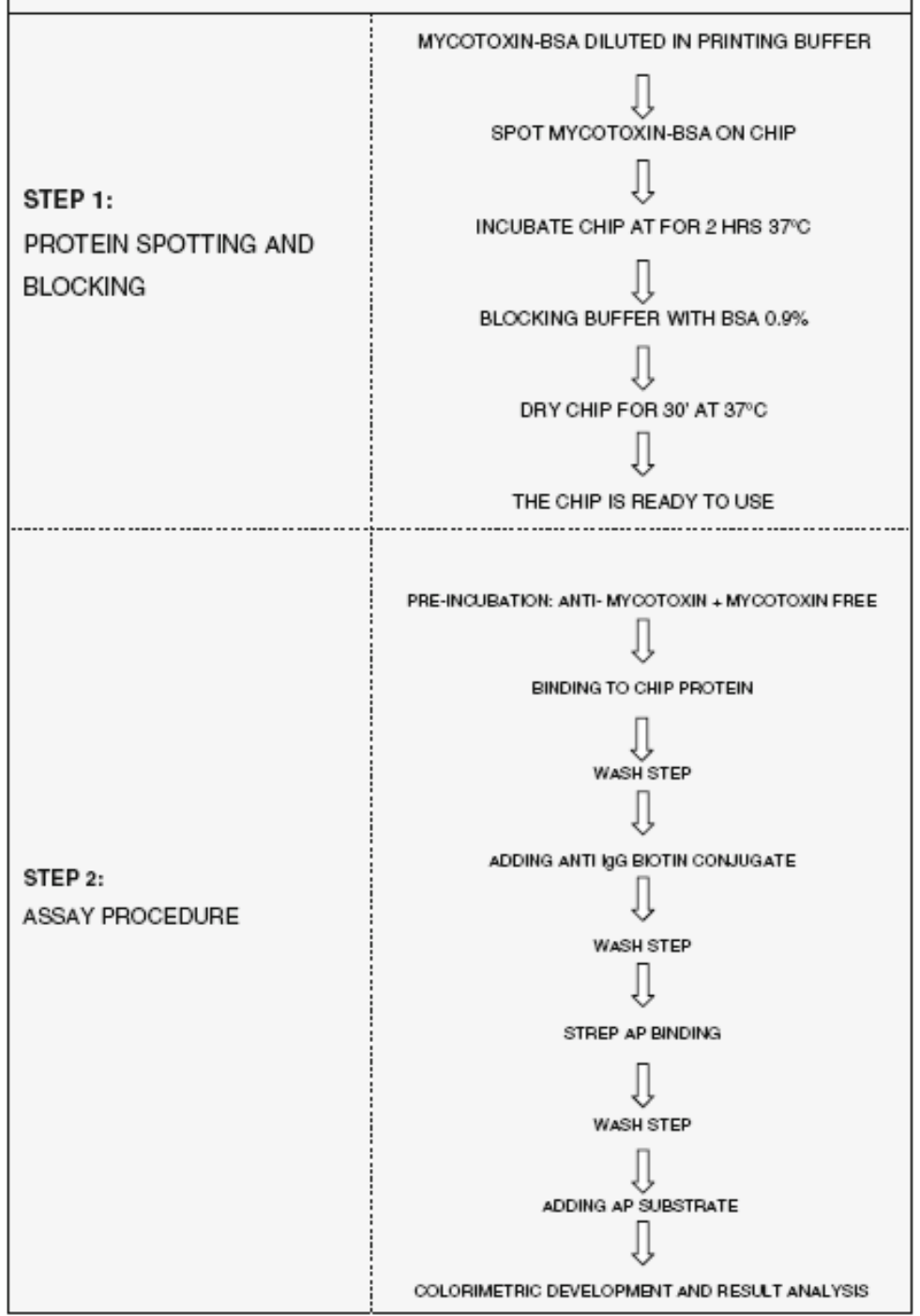

Fig. 7. Scheme of sensing assay for Aflatoxin B1 (Lamberti et al., 2009)

\section{Conclusion}

Mycotoxin analysis in food and feed is generally a multi-step process comprised of sampling, sample preparation, toxin extraction from the matrix (usually with mixtures of water and polar organic solvents), extract clean-up and finally detection and quantitative determination, for these reasons. 
The availability of rapid and reliable methods for "in field" determination of fungine contamination, as Aflatoxins and other MTXs (mycotoxins) identification in foods is an increasing need for human health and food safety purposes. Gas chromatographic (GC) methods for Aflatoxins detection are generally used, due to their low detection limits and high selectivity. Laboratories generally have to analyze a large number of samples requiring adequate storage conditions and time-consuming sample pre-treatment and preconcentration procedures. The establishment of efficient method for Aflatoxins detection is therefore of high importance and new trends consist in development portable and easy to use biosensors.

Recently, biosensors have seen a great development in the field of small molecules analytical determination and these methods are upon constant improvement also for MTXs and for Aflatoxins, but actually generally restricted to feasibility studies.

At this developmental stage, biosensors for Aflatoxins and MTX (Mycotoxin) detection could be very useful as a qualitative/semiquantitative "field test" for identifying "positive" samples, reducing the number of samples to be reanalyzed in the laboratory, according to analytical standard methods (GC).

Biosensor assays are rapid, easy to perform, and inexpensive and could be advantageous in comparison with ELISA or GC/MS for analysis on food, but in our opinion, further improvements of analytical parameters such as precision, accuracy, and detection limits (especially for Aflatoxins biosensor applications) are required.

\section{Acknowledgment}

The authors are grateful to Prof.Tibor Hianik of Nuclear Physics and Biophysics, Comenius University, Bratislava, Slovakia and to Prof.Caterina Tanzarella, University of RomaTre Department of Biology, Rome, Italy for their advice and scientific support.

\section{References}

Alonso-Lomillo, M.A., Domínguez-Renedo, O., Ferreira-Gonçalves, L., \& Arcos-Martínez, M.J. (2010). Sensitive enzyme-biosensor based on screen-printed electrodes for Ochratoxin A. Biosensors and Bioelectronics, Vol. 25, No 6, pp. 1333-1337, 09565663,

Blohm, D.H., \& Guiseppi-Elie, A. (2001). New developments in microarray technology. Current Opinion in Biotechnology, Vol. 12, No 1, pp. 41-47, 0958-1669.

Cruz-Aguado, J.A., \& Penner, G. (2008). Determination of ochratoxin A with a DNA aptamer. Journal of Agricultural and Food Chemistry, Vol. 56, No 22, pp. 10456-10461, 0021-8561,.

Cuccioloni, M., Mozzicafreddo, M., Barocci, S., Ciuti, F., Pecorelli, I., Eleuteri, A.M., Spina, M., Fioretti, E., \& Angeletti, M. (2008). Biosensor-based screening method for the detection of aflatoxins B 1-G1. Analytical Chemistry, Vol. 80, No 23, pp. 9250-9256, 0003-2700

Ehrlich, K.C., Montalbano, B.G., \& Cotty, P.J. (2003). Sequence comparison of aflR from different Aspergillus species provides evidence for variability in regulation of Aflatoxin production. Fungal Genetics and Biology Vol. 38, pp. 63-74, 1087-1845.

Gagliardi, S., Rapone, B., Mosiello, L., Luciani, D., Gerardino, A., \& Morales, P. (2007). Laserassisted fabrication of biomolecular sensing microarrays, IEEE Transactions on Nanobioscience, Vol. 6, No 3, pp. 242-248, 1536-1241. 
Homola J., 2008, Surface Plasmon Resonance Sensors for Detection of Chemical and Biological Species, Chemical Review, Vol. 108, 462-493, 0009-2665.

Ko, S., Kim, C.J., \& Kwon, D.Y. (2010). Detection of aflatoxin B1 by SPR biosensor using fusion proteins as a linker. Proceedings of the 2010 NSTI Nanotechnology Conference and Expo, 978-1439834183, Anaheim, 978-1-4398-3418-3, CA, June 2010.

Koppal, T. (2004) Microarrays: migrating from discovery to diagnostics. Drug Discovery Development, Vol. 7, pp. 30-34.

Lamberti I., Tanzarella C., Solinas I., Padula C. \& Mosiello L. (2009). An antibody-based microarray assay for the simultaneous detection of aflatoxin B1 and fumonisin B1. Mycotoxin Research, Vol. 25 No 4, pp. 193-200, 0178-7888.

Lamberti, I., Mosiello, L., Cenciarelli, C., Antoccia, \& A., Tanzarella, C. (2010). A novel based protein microarray for the simultaneous analysis of activated caspases. Lecture Notes in Electrical Engineering, Vol. 54, pp. 323-326, 1876-1100.

Lamberti, I., Mosiello L., \& Hianik, T. (2011). Development of thickness shear mode biosensor based on DNA aptamers for detection ochratoxin. Chemical Sensors, Vol 1, pp. 11-15, 2231-6035.

Li, S.C., Chen, J.H., Cao, H., Yao, D.S., \& Liu, D.L. (2011). Amperometric biosensor for aflatoxin B1 based on aflatoxin-oxidase immobilized on multiwalled carbon nanotubes. Food Control, Vol. 22, No 1, pp. 43-49, 0956-7135.

Oliveira, S.C.B., Diculescu, V.C., Palleschi, G., Compagnone, D., \& Oliveira-Brett, A.M. (2007). Electrochemical oxidation of ochratoxin A at a glassy carbon electrode and in situ evaluation of the interaction with deoxyribonucleic acid using an electrochemical deoxyribonucleic acid-biosensor. Analytica Chimica Acta, Vol. 588, No 2, pp. 283-291, 0003-2670.

Otim, M.O., Mukiibi-Muka, G., Christensen, H. \& Bisgaard, M. (2005). Aflatoxicosis, infectious bursal disease and immune response to Newcastle disease vaccination in rural chickens. Avian Pathology Vol. 34: pp. 319-323, 0307-9457.

Piermarini, S., Volpe, G., Ricci, F., Micheli, L., Moscone, D., Palleschi, G., Führer, M., Krska, R., \& Baumgartner, S. (2007). Rapid screening electrochemical methods for aflatoxin B1 and type-A trichothecenes: A preliminary study. Analytical Letters, Vol. 40 No 7, pp. 1333-1346, 0003-2719.

Prieto-Simón, B., Noguer, T., \& Campàs, M. , 2007. Emerging biotools for assessment of mycotoxins in the past decade. TrAC. Trends in Analytical Chemistry, Vol. 26 No 7, pp. 689-702, 0165-9936.

Siontorou, C.G., Nikolelis, D.P., Miernik, A., \& Krull, U.J. (1998). Rapid methods for detection of Aflatoxin M1 based on electrochemical transduction by self-assembled metal-supported bilayer lipid membranes (s-BLMs) and on interferences with transduction of DNA hybridization. Electrochimica Acta, Vol 43 No23, pp. 3611-3617, 0013-4686.

Tsai, W.-C., \& Hsieh, C.-K. (2007). QCM-based immunosensor for the determination of ochratoxin A. Analytical Letters, Vol. 40 No 10, pp. 1979-1991.

Turner, N.W., Subrahmanyam, S., \& Piletsky, S.A. (2009). Analytical methods for determination of mycotoxins: A review. Analytica Chimica Acta, Vol. 632 No 2, pp. 168-180, 0003-2670. 
Turner, P.C., Moore, S.E., Hall, A.J., Prentice, A.M. \& Wild, C.P. (2003). Modification of immune function through exposure to dietary aflatoxin in Gambian children. Environmental Health Perspectives, Vol. 111, pp. 217-220.

Ueno Y, Iijima K, Wang S-D, Sugiura Y, Sekijima M, Tanaka T, Chen C, \& Yu S-Z. (1997). Fumonisin as a possible contributory risk factor for primary liver cancer: a 3-year study of corn harvested in Haimen, China by HPLC and ELISA. Food and Chemical Toxicology Vol. 35, pp. 1143-1150, 0278-6915.

Valdivia, A.G., Martinez, A., Damian, F.J., Quezada, T., Ortiz, R., Maryinez, C., Llamas, J., Rodriguez, M.L., Yamamoto, L., Jaramillo, F., Loarca-Pina, M.G. \& Reyes, J.L. (2001). Efficacy of N-acetylcysteine to reduce the effects of aflatoxin B1 intoxication in broiler chickens. Poultry Science, Vol 80, pp. 727-734, 0032-5791.

Van Der Merwe, K.J., Steyn, P.S., Fourie, L., Scott, D.B., Theron, J.J. (1965). Ochratoxin A, a toxic metabolite produced by Aspergillus ochraceus Wilh Nature, Vol. 205, No 4976, pp. 1112-1113.

Venkatasubbarao, S. (2004). Microarrays - status and prospects. Trends in Biotechnology, Vol. 22 No12, pp. 630-637, 0167-7799. 


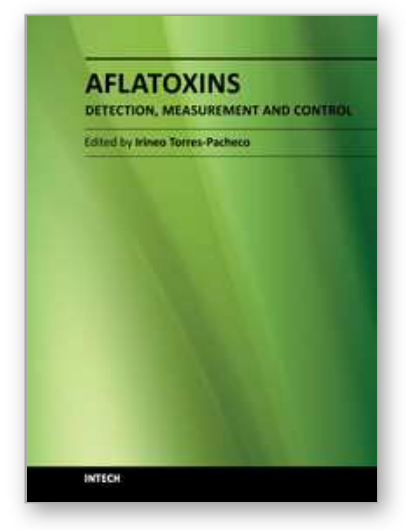

\author{
Aflatoxins - Detection, Measurement and Control \\ Edited by Dr Irineo Torres-Pacheco
}

ISBN 978-953-307-711-6

Hard cover, 364 pages

Publisher InTech

Published online 21, October, 2011

Published in print edition October, 2011

This book is divided into three sections. The section called Aflatoxin Contamination discusses the importance that this subject has for a country like the case of China and mentions examples that illustrate the ubiquity of aflatoxins in various commodities The section Measurement and Analysis, describes the concept of measurement and analysis of aflatoxins from a historical prespective, the legal, and the state of the art in methodologies and techniques. Finally the section entitled Approaches for Prevention and Control of Aflatoxins on Crops and on Different Foods, describes actions to prevent and mitigate the genotoxic effect of one of the most conspicuous aflatoxins, AFB1. In turn, it points out interventions to reduce identified aflatoxin-induced illness at agricultural, dietary and strategies that can control aflatoxin. Besides the preventive management, several approaches have been employed, including physical, chemical biological treatments and solvent extraction to detoxify AF in contaminated feeds and feedstuffs.

\title{
How to reference
}

In order to correctly reference this scholarly work, feel free to copy and paste the following:

Lucia Mosiello and llaria Lamberti (2011). Biosensors for Aflatoxins Detection, Aflatoxins - Detection, Measurement and Control, Dr Irineo Torres-Pacheco (Ed.), ISBN: 978-953-307-711-6, InTech, Available from: http://www.intechopen.com/books/aflatoxins-detection-measurement-and-control/biosensors-for-aflatoxinsdetection

\section{INTECH}

open science | open minds

\section{InTech Europe}

University Campus STeP Ri

Slavka Krautzeka 83/A

51000 Rijeka, Croatia

Phone: +385 (51) 770447

Fax: +385 (51) 686166

www.intechopen.com

\section{InTech China}

Unit 405, Office Block, Hotel Equatorial Shanghai

No.65, Yan An Road (West), Shanghai, 200040, China

中国上海市延安西路65号上海国际贵都大饭店办公楼 405 单元

Phone: +86-21-62489820

Fax: $+86-21-62489821$ 
(C) 2011 The Author(s). Licensee IntechOpen. This is an open access article distributed under the terms of the Creative Commons Attribution 3.0 License, which permits unrestricted use, distribution, and reproduction in any medium, provided the original work is properly cited. 